\title{
Deciphering post-infarct inflammation: Should it heal, would it hurt?
}

\author{
Matthias Nahrendorf, PhD, ${ }^{a}$ Antonio Abbate, $\mathrm{MD}, \mathrm{PhD},{ }^{\mathrm{b}}$ \\ and Jagat Narula, MD, PhD, MACC, MASNC \\ a Massachusetts General Hospital, Harvard Medicial School, Boston, MA \\ b VCU Pauley Heart Center, Virginia Commonwealth University, Richmond, VA \\ c Mount Sinai Morningside Hospital, Icahn School of Medicine at Mount Sinai, New York, NY
}

Received Jan 22, 2020; accepted Jan 22, 2020

doi: $10.1007 / \mathrm{s} 12350-020-02053-\mathrm{z}$

\section{See related article, pp. 2083-2099}

Technology development in phenotypic characterization has played a catalytic role in biomedical research and has allowed superior understanding of pathogenesis, diagnosis, and management of cardiovascular diseases. In their review in this issue of the Journal of Nuclear Cardiology, Wilk et al. ${ }^{1}$ describe the promise of marrying two sophisticated clinical imaging modalities that rely on unrelated detection strategies-positron emission tomography and magnetic resonance imaging. The combination of these two powerful technologies, already largely employed jointly in the management of patients of cancer, are equipped to open new vistas into cardiovascular pathology, as outlined by the state-of-art review that focusses on imaging myocardial inflammation after acute myocardial infarction (AMI). Among various cardiovascular diseases, the choice of acute myocardial infarction is logical, relatively easily accessible, and likely to accrue substantial gains.

AMI leads to highly dynamic immune system adaptions $^{2}$, with large imaging biomarker deviations from the steady state and important consequences for subsequent disease trajectories (Figure 1). The most

Reprint requests: Jagat Narula, MD, PhD, MACC, MASNC, Mount Sinai Morningside Hospital, Icahn School of Medicine at Mount Sinai, 421 W. 113th Street, Suite 130, New York, NY 10025; narula@mountsinai.org

J Nucl Cardiol 2020;27:2100-2.

$1071-3581 / \$ 34.00$

Copyright (C) 2020 American Society of Nuclear Cardiology, corrected publication 2020 numerous leukocytes that propagate inflammation in the heart are macrophages. Even the healthy heart contains a sizable population of macrophages; they comprise $7 \%$ $8 \%$ of the non-cardiomyocytes. These macrophages are heart-specific, differ from macrophages that reside in other organs and evolve from local proliferation in the heart. Their subsets do not resemble M1/M2 phenotypes reported in vitro ${ }^{3}$. The nomenclature currently established describes four macrophage subsets identified by the surface markers MHCII and CCR $2^{4}$, with additional markers emerging. The heterogeneity of macrophages is a rapidly expanding discipline, as single cell RNA sequencing studies suggest that many more than just these four subsets are likely present ${ }^{5}$. Ongoing work is striving to define macrophage subset-specific functions, as only deciphering specific function would make identifying cell subsets meaningful.

In AMI, the resident macrophage population dies quickly alongside ischemic cardiomyocytes ${ }^{6}$. Subsequently, the ischemic wound recruits monocytes from the blood, which are produced in the bone marrow and spleen $^{2}$. This is an important switch in cell origin which may facilitate the dynamic changes in macrophage function over the next days, when a constantly replenishing ensemble of leukocytes is hard at work repairing the infarct for up to 2 weeks. The phenotypes of these cells are in flux and become less inflammatory as time progresses. We propose to describe these phenotypes as "inflammatory macrophages" early on and "reparatory macrophages" in later stages. Macrophages may play a reparatory role initially by modulating the number and function of the recruited fibroblasts and subsequently more directly by converting into fibroblast-like cells and depositing collagen and extracellular matrix ${ }^{7,8}$. The newly converted fibroblasts do not express many fibroblast markers of conventional fibroblasts. Transcription factor KLF4 has been shown to facilitate 


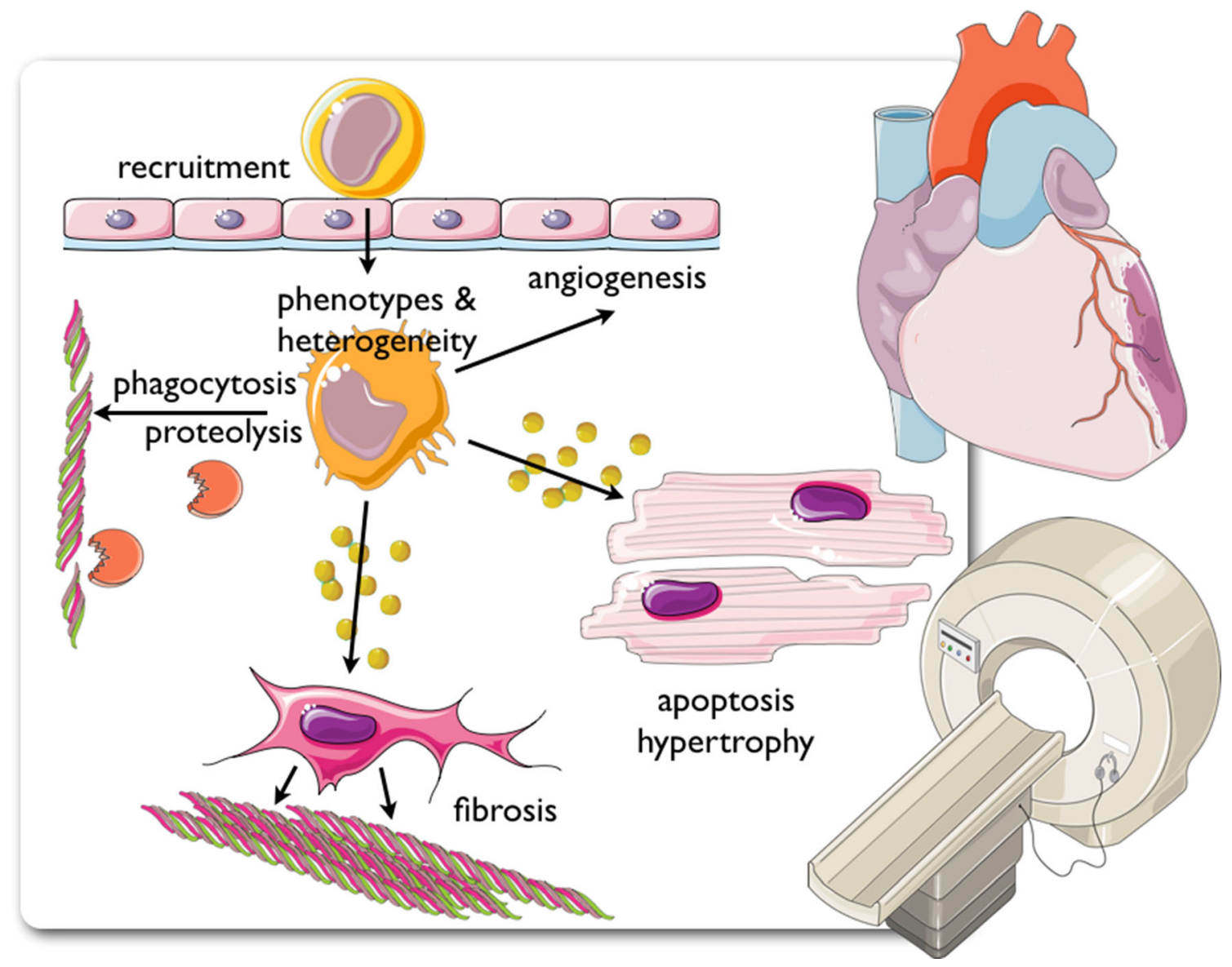

Figure 1. PET-MRI is poised to report not only on cardiac anatomy and function but also on molecular processes that determine recovery from AMI, including inflammation, fibrosis, and angiogenesis.

phenotypic transition, and its up-regulation during the healing phase of mouse myocardium in $\mathrm{CD} 90^{+} \mathrm{F} 4 / 80^{+}$ cells indicates an ongoing macrophage to fibroblast transition.The newly converted pathogenic fibroblasts perhaps lack the control, competence, and finesse of a conventional fibroblast in maintaining collagen homeostasis and may engage in uncontrolled deposition of collagen and extracellular matrix.

Understandably so, therapeutic strategies are being explored aggressively aimed at modulating inflammation during AMI. In the recently presented COLCOT study, colchicine-a potent antiinflammatory medication that is indicated for the treatment of gout and pericarditis, at a small daily dose led to a significantly lower percentage of post-infarct patients with ischemic cardiovascular events than placebo. ${ }^{9}$ The benefits of colchicine with regard to cardiovascular end points in COLCOT were at least as large as those of canakinumab in CANTOS, wherein interleukin-1 $\beta$ (IL-1 $\beta$ ) blockade was investigated. ${ }^{10}$ The use of experimental parabiosis surgery in animals has shown that IL- $1 \beta$ increase bone marrow hematopoietic stem cell proliferation after AMI and contributes to myocardial inflammation leading to heart failure after $\mathrm{AMI}^{11}$. A clinical trial with an IL-1 blocker, anakinra, showed prevention of heart failure in patients with ST-segment elevation AMI when treated for 14 days during the index event ${ }^{12}$. Whether and how the treatment affected the recruitment of inflammatory cells within the heart of the patients with AMI remains unexplored.

While our insight into the complex inflammatory processes after AMI expands due to single cell diagnostics, the current clinical tools fall short of providing any such insight. Mostly, we are limited to blood biomarkers such as C-reactive protein and differential leukocyte counts. Given the multitude of processes that regulate the number and heterogeneity of leukocytes in tissue, including recruitment, differentiation, phagocytosis and apoptosis, blood biomarkers provide an incomplete picture of what happens in the heart. To explicitly understand the local events in diseased tissue and how to best optimize them therapeutically, we would need time-resolved quantification of leukocyte numbers, subsets, and cell functions such as removal of 
tissue and instigation of fibrosis. Such complex in vivo imaging is very difficult to achieve, even in the mouse. In preclinical systems, the versatility of fluorescence imaging has pushed the number of simultaneously and non-invasively sampled imaging biomarkers to four. This is currently not possible in patients, however, PETMRI expands these capabilities to two independent molecular or cellular biomarkers-a big step forward for clinical imaging. The most exciting applications for this modality will enable sampling two inflammation-related, orthogonal biomarkers, for instance the number of macrophages (using PET isotope labeled nanoparticles) and their predominant phenotype (using smart MRI agents such as MPO-Gd) ${ }^{10}$. Such hybrid data sets will inform whether there is an oversupply of leukocytes, whether inflammatory cell phenotypes persist for too long, or both; and could trigger interventions tailored to the specific situation and patient. Such imaging scenarios are currently unavailable clinically, and may be unaffordable for large-scale adoption once they reach clinical translation. However, non-invasive, dual imaging and molecular imaging will no doubt play important roles in translating preclinical discoveries and in clinical trials. Following on the heels of the immunotherapy revolution in oncology, similar breakthroughs are on the horizon for cardiovascular patients. Imaging should play an important role there, as an end point for intermediate trials before moving on to launching vastly expensive studies with mortality endpoints. We believe that combining PET with MRI has only started to demonstrate its potential, ${ }^{13}$ and that clinical research applications will greatly benefit from the merger of two imaging technologies.

\section{Disclosures}

Dr Nahrendorf has obtained research grants from NIH, $M G H$ and AHA. Dr Abbate has received research grants from Swedish Orphan Biovitrum (Stockholm, Sweden). Dr Narula has revealed no conflicts of interest.

\section{References}

1. Wilk B, Wisenberg G, Dhamakumar R, Thiessen JD, Goldhawk DE, Prato FS. Hybrid PET/MR imaging in myocardial inflammation post-myocardial infarction. J Nucl Cardiol 2019. https://d oi.org/10.1007/s12350-019-01973-9.

2. Nahrendorf M. Myeloid cell contributions to cardiovascular health and disease. Nat Med 2018;24:711-20.

3. Nahrendorf M, Swirski FK. Abandoning M1/M2 for a network model of macrophage function. Circ Res 2016;119:414-7.

4. Epelman S, Lavine KJ, Randolph GJ. Origin and functions of tissue macrophages. Immunity 2014;41:21-35.

5. Dick SA, Macklin JA, Nejat S, Momen A, Clemente-Casares X, Althagafi MG, et al. Self-renewing resident cardiac macrophages limit adverse remodeling following myocardial infarction. Nat Immunol 2019;20:29-39.

6. Leuschner F, Rauch PJ, Ueno T, Gorbatov R, Marinelli B, Lee WW, et al. Rapid monocyte kinetics in acute myocardial infarction are sustained by extramedullary monocytopoiesis. J Exp Med 2012;209:123-37

7. Haider N, Boscá L, Zandbergen HR, Kovacic JC, Narula N, González-Ramos S, et al. Transition of macrophages to fibroblastlike cells in healing myocardial infarction. J Am Coll Cardiol 2019;74:3124-35

8. Libby P, Swirski FK, Nahrendorf M. The myocardium: More than myocytes. J Am Coll Cardiol 2019;74:3136-8.

9. Tardif JC, Kouz S, Waters DD, Bertrand OF, Diaz R, Maggioni $\mathrm{AP}$, et al. Efficacy and safety of low-dose colchicine after myocardial infarction. N Engl J Med 2019;381:2497-505.

10. Ridker PM, Everett BM, Thuren T, MacFadyen JG, Chang WH, Ballantyne C, et al. Antiinflammatory therapy with canakinumab for atherosclerotic disease. N Engl J Med 2017;377:1119-31.

11. Sager HB, Heidt T, Hulsmans M, Dutta P, Courties G, Sebas M, et al. Targeting interleukin- $1 \beta$ reduces leukocyte production after acute myocardial infarction. Circulation 2015;17:1880-90.

12. Abbate A, Trankle CR, Buckley L, Lipinski MJ, Appleton D, Kadariya D, et al. Interleukin-1 blockade Inhibits the acute inflammatory response in patients with ST-segment elevation myocardial infarction. J Am Heart Assoc 2020; in press.

13. Vandoorne K, Rohde D, Kim HY, Courties G, Wojtkiewicz G, Honold L, et al. Imaging the vascular bone marrow niche during inflammatory stress. Circ Res 2018;123:415-27.

Publisher's Note Springer Nature remains neutral with regard to jurisdictional claims in published maps and institutional affiliations. 